\title{
Y-39983 downregulates RhoA/Rho-associated kinase expression during its promotion of axonal regeneration
}

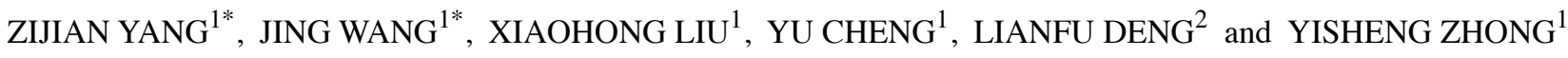 \\ ${ }^{1}$ Department of Ophthalmology, Ruijin Hospital Affiliated Medical School, Shanghai Jiaotong University; \\ ${ }^{2}$ Shanghai Institute of Traumatology and Orthopedics, Shanghai 200025, P.R. China
}

Received October 23, 2012; Accepted December 5, 2012

DOI: 10.3892/or.2012.2205

\begin{abstract}
Y-39983, a selective Rho-associated kinase (ROCK) inhibitor, promotes axonal regeneration of damaged retinal ganglion cells (RGCs). The present study investigated the effects of Y-39983 on RhoA/ROCK expression during promotion of axonal regeneration using a rat optic nerve crush (ONC) model. Herein, we demonstrated that Y-39983 significantly enhanced the survival and axonal regeneration of RGCs after ONC. Using a pull-down assay and affinity precipitation to examine the activity of RhoA, we detected the decreased expression of active-RhoA after using Y-39983. The expression of ROCK1 and ROCK2 was significantly decreased as demonstrated by RT-PCR, immunohistochemistry and western blot analysis. The downregulation of active-RhoA, ROCK1 and ROCK2 expression by Y-39983 coincided with the appearance of larger numbers of regenerating axons. In conclusion, Y-39983 downregulated the expression of activeRhoA, ROCK1 and ROCK2 during its promotion of axonal regeneration.
\end{abstract}

\section{Introduction}

Axonal regeneration depends on the environment and the intrinsic regenerative capacity of neurons in the adult central nervous system (CNS). After CNS injury, the regenerative ability is reduced in comparison to embryonic neurons, which is partially reflected in the differential regulation of regenera-

Correspondence to: Dr Yisheng Zhong, Department of Ophthalmology, Ruijin Hospital Affiliated Medical School, Shanghai Jiaotong University, 197 Ruijin No. 2 Road, Shanghai 200025, P.R. China

E-mail: yszhong68@yahoo.com.cn

Dr Lianfu Deng, Shanghai Institute of Traumatology and Orthopedics, 197 Ruijin No. 2 Road, Shanghai 200025, P.R. China

E-mail: lfdeng@msn.com

${ }^{*}$ Contributed equally

Key words: Y-39983, retinal ganglion cells, axonal regeneration, RhoA/Rho-associated kinase tion-associated proteins, such as growth associated protein-43 (GAP-43) $(1,2)$. In the adult CNS, poor axonal regeneration of injured neurons is in part due to the existence of myelinassociated axonal growth inhibitors such as myelin-associated glycoprotein (MAG), Nogo and oligodendrocyte-myelin glycoprotein $(\mathrm{OMgp})(3,4)$. These inhibitors activate RhoA, a member of the small GTPases, which is known to regulate the actin cytoskeleton $(5,6)$. RhoA acts as a molecular switch that controls various intracellular signaling pathways by changing between an active (GTP-bound) and inactive (GDP-bound) form. Several studies have shown that the activation of RhoA results in growth cone collapse and neurite outgrowth inhibition, and the inactivation of RhoA could promote axonal regeneration and functional recovery in the injured CNS (7-9). The downstream effector of RhoA is the Rho-associated kinase (ROCK), including ROCK1 and ROCK2 $(10,11)$. Activation of ROCK1 and ROCK2 enhances phosphorylation of the regulatory myosin light-chain phosphatase (MLCP), which is a key step in cytoskeletal rearrangement (12). The activity of ROCK1 and ROCK2 is inhibited by ROCK inhibitor Y-27632 $(13,14)$ and inactivation of ROCK can promote axonal growth $(8,15)$.

Y-39983, a selective ROCK inhibitor, has great potency at inhibiting the activity of ROCK1 and ROCK2 (16). Previous studies have shown that Y-39983 promotes axonal regeneration of damaged retinal ganglion cells (RGCs) $(15,17,18)$. Despite the demonstrated benefits in neuro-regeneration, there are no data available concerning the effects of Y-39983 on RhoA/ ROCK expression during its promotion of axonal regeneration. Therefore, the present study investigated the effects of Y-39983 on RhoA/ROCK expression in the promotion of axonal regeneration of RGCs.

\section{Materials and methods}

Animals. Adult male Sprague-Dawley rats (200-250 g) were obtained from the Laboratory Animal Center of Shanghai Institute of Traumatology and Orthopedics, Shanghai Jiaotong University, China. They were housed individually and maintained on a 12-h light/dark cycle. Food and water were available ad libitum. All experiments were performed in accordance with the Association for Research in Vision and Ophthalmology (ARVO) Statement for the Use of Animals in Ophthalmic and Vision Research. Animals were anesthetized using a $10 \%$ chloral hydrate solution $(420 \mathrm{mg} / \mathrm{kg}$ b.w.), and 
sacrificed using an intraperitoneal overdose of a $30 \%$ solution. Every effort was made to minimize suffering and limit the number of animals used.

Retrograde labeling of RGCs. To determine densities of the RGCs, all experimental rats were randomly chosen for the retrograde labeling of RGCs by fluorescent tracer DiI. The retrograde labeling of RGCs was performed as previously described (19). Briefly, animals were anesthetized, the skin was incised mediosagitally, and the skull cartilage was opened dorsal to the lambda fissure. DiI (1.5 $\mu \mathrm{l})(10 \%$ in dimethylsulfoxide) was then applied to both superior colliculi (SC), respectively, using a micropipette. The optic nerve crush was performed as described below 3 days after DiI labeling.

Optic nerve crush (ONC) model. Surgical procedures were similar to those described previously (20). Rats were anesthetized, and a 1- to $1.5-\mathrm{cm}$ incision was made in the skin above the right orbit. The optic nerve (ON) of the right eye was exposed under an operating microscope, and the sheath was opened longitudinally. Using special forceps (40 g), the ON was crushed within the sheath at the site $1 \mathrm{~mm}$ behind the optic nerve head for $9 \mathrm{sec}$, avoiding injury to the ophthalmic artery. Nerve injury was verified by the appearance of a clearing at the crush site; the vascular integrity of the retina was verified by fundoscopic examination after dilating the pupil with tropicamide. In the sham operation group, the $\mathrm{ON}$ of the right eye was exposed, and the sheath was opened longitudinally, but without the crush procedure.

Drug injection into the vitreous. The drugs were administered to the vitreous via a stereotactically positioned 30-gauge needle attached to a $10-\mu 1$ Hamilton syringe. A final volume of $3 \mu \mathrm{l}$ was administered via the peripheral temporal retinal site taking care to avoid contact with the lens since its injury releases neuroregenerative factors (21). The eye was examined ophthalmoscopically to check that the retinal vasculature was intact. Y-39983 (4-[(1R)-aminoethyl]- $N$-(1H-pyrrolo[2,3-b] pyridine-4-yl)benzamide monohydrochloride), ( $\mathrm{MW}=316.8$; Tocris Bioscience, Ellisville, MO, USA) was diluted in phosphate-buffered saline (PBS). According to the drug administration, the animals were divided into three groups: the sham-operated group (sham group), the ONC+PBS intravitreal injection group (ONC+PBS group) and the ONC+ Y-39983 intravitreal injection group (ONC+Y-39983 group). Apart from the sham group while received no intravitreal injection, the other groups received $3 \mu \mathrm{l}$ PBS or $20 \mu \mathrm{M}$ [intraocular end density as mean vitreous volume $56 \mu 1$ (22)] Y-39983 intravitreal injection, respectively, immediately after ONC. The doses of Y-39983 used in the present study were chosen according to a previous study (18). The second injection of $3 \mu \mathrm{l}$ PBS or $20 \mu \mathrm{M}$ Y-39983 was performed on day 7 after ONC. Groups of rats were sacrificed 15 days after surgery.

Sample preparations of the retina and optic nerves. Fifteen days after ONC, deeply anesthetized rats were transcardially perfused with $4 \%$ paraformaldehyde. For mRNA analysis, the rats were directly sacrificed by an intraperitoneal overdose of anesthetic without transcardial perfusion to avoid mRNA degradation. The eyes were enucleated with at least $5 \mathrm{~mm}$ of optic nerve attached and bisected. The eyes were dissected as eye cups without cornea and lens; the optic nerves were dissected free from connective tissue. Retinas were dissected from the underlying sclera for further use. The optic nerves were immediately fixed overnight, then transferred to $30 \%$ sucrose solution overnight $\left(4^{\circ} \mathrm{C}\right)$, and the frozen sections were cut. Optic nerve longitudinal sections $(16 \mu \mathrm{m})$ were cut along the optic nerve anterior-posterior axis. The sections were collected on gelatin-coated glass slides and stored at $-80^{\circ} \mathrm{C}$ for further use.

$R G C$ densities. Retinas were dissected from the underlying sclera, flattened by four radial cuts, and mounted vitreal side-up on gelatin-coated slides. Labeled RGCs were examined by a confocal microscope (Axiovert 35; Zeiss, Germany) using a rhodamine filter (560 $\mathrm{nm}$ for DiI). RGC densities were determined by counting labeled RGCs in 12 distinct areas of $62,500 \mu \mathrm{m}^{2}$ each (four areas per retinal quadrant at three different eccentricities of $1 / 6,3 / 6$ and $5 / 6$ of the retinal radius). Cell counting was performed with a computerized image-analysis system (Image Pro Plus version 6.0; Media Cybernetics, Silver Spring, MD, USA) in duplicate by two independent investigators in a blinded manner. The averages of the number of RGCs in 12 distinct areas were taken as the mean density of RGCs for each retina. Eighteen rat retinas were used for RGC densities analysis and there were six rats in each group.

Immunohistochemistry. Optic nerve sections were dehydrated at $37^{\circ} \mathrm{C}$ for $1 \mathrm{~h}$, and unspecific binding was blocked by application of $10 \%$ normal goat serum. Primary antibodies including GAP-43 monoclonal antibody (1:100, sc-17790), ROCK1 monoclonal antibody (1:50, sc-17794), ROCK2 polyclonal antibody (1:50, sc-5561) (all from Santa Cruz Biotechnology, Santa Cruz, CA, USA) were applied at $4^{\circ} \mathrm{C}$ overnight. Negative controls were performed by replacing the primary antibody with PBS or serum. Secondary antibodies including $\mathrm{Cy}$-3-labeled anti-rat IgG, FITC-labeled anti-rabbit or anti-mouse antibody (all from Invitrogen, CA, USA) were applied (1:100) for $45 \mathrm{~min}$ at room temperature. Cell nuclei were counterstained with DAPI (4',6-diamidino2-phenylindole) (1:1000; Sigma-Aldrich, St. Louis, MO, USA). Immunoreactivity was examined with a confocal microscope (Axiovert 35, Zeiss, Germany). For evaluation of axon regeneration of RGCs, counting of the GAP-43-positive axons was performed as previously described (23). Briefly, the optic nerve sections with anti-GAP-43 immunoreactivity were photographed using a confocal microscope. Images of whole sections were assembled from single images captured with a x20 objective. Using a calibrated ocular to measure distance, we counted the number of GAP-43-positive axons crossing a line at distance $d(50,250,500 \mu \mathrm{m})$ from the end of the crush site. By measuring the cross-sectional width of the nerve at the point at which the counts were taken, we converted axon counts into axon crossings per unit nerve width (axons per millimeter) and obtained the average of these over the four sections. $\sum \mathrm{a}_{d}$, the total number of axons extending distance $d$ in a nerve having a radius of $r$, was estimated by summing over all sections of thickness $t$. For all immunohistochemical 
staining, three sections per eye were examined and there were six rats in each group.

In situ active-RhoA pull-down assay. Active-RhoA pull-down assay was performed according to a modified previous published method $(24,25)$. Briefly, $16-\mu \mathrm{m}$ frozen sections of optic nerve were post-fixed in $4 \%$ paraformaldehyde and incubated with glutathione-S-transferase (GST)-Rho-binding domain (RBD) fushion protein (14-662; Millipore, Billerica, MA, USA) overnight at $4^{\circ} \mathrm{C}$. Sections were then washed 3 times in PBS, blocked in $10 \%$ normal goat serum for $1 \mathrm{~h}$ at room temperature and incubated with an anti-GST antibody (Millipore) overnight at $4^{\circ} \mathrm{C}$. Sections were then washed in PBS, incubated with $\mathrm{Cy}$-3-labeled anti-rat secondary antibodies (Invitrogen). The specimens were imaged with a confocal microscope (Axiovert 35, Zeiss). Three sections per eye were examined and there were six rats in each group.

Reverse transcription-polymerase chain reaction (RT-PCR). Twenty-four rat retinas were used for RT-PCR analysis. Each retina served as an individual sample ( $n=8$ per group). Total RNA was isolated from the pulverized samples using the TRIzol $^{\circledR}$ reagent (Invitrogen). The concentration and purity of the preparations were determined by measuring the absorbance at 260/280 $\mathrm{nm}$ in a spectrophotometer (Beckman). Total RNA was reverse-transcribed into cDNA in a $20-\mu 1$ reaction containing $2 \mu \mathrm{g}$ RNA, $4 \mu \mathrm{l}$ 5X M-MLV buffer, $2 \mu \mathrm{l}$ of dNTP, $1 \mu \mathrm{l}$ random hexamer primer, $0.5 \mu \mathrm{l}$ of RNase inhibitor and $1 \mu \mathrm{l}$ of M-MLV RTase. Reactions were performed at $25^{\circ} \mathrm{C}$ for $10 \mathrm{~min}$, at $42^{\circ} \mathrm{C}$ for $60 \mathrm{~min}$ and at $70^{\circ} \mathrm{C}$ for $10 \mathrm{~min}$.

The following targets were analyzed by RT-PCR. The nucleotide sequences of the primers were based on previously published sequences $(26,27)$. The primer sequences used for RT-PCR were: RhoA, 5'-GTGATTGTTGGTGATGGAGC-3' and 5'-CTCGTGGCCATCTCAAAAAC-3'; ROCK-1, 5'-TGC GGGAGTTACAAGATCAGCT-3' and 5'-TTTCCGTCA GTCTCATCAGCAC-3'; ROCK-2, 5'-TCTGAAAGGAGG GACCGAACC-3' and 5'-GTTCCTGTTTGTGTCGAGCCA TCA-3'; glyceraldehyde 3-phosphate dehydrogenase (GAPDH), 5'-ATGGGGAAGGTGAAGGTCGG-3' and 5'-CAGGAGG CATTGCTGATGAT-3'. The PCR protocol comprised an initial incubation for $5 \mathrm{~min}$ at $94^{\circ} \mathrm{C} ; 30$ cycles (for RhoA, ROCK-1 and ROCK-2) or 25 cycles (for GAPDH) of $45 \mathrm{sec}$ at $94^{\circ} \mathrm{C}, 45 \mathrm{sec}$ at $55^{\circ} \mathrm{C}$, and $2 \mathrm{~min}$ at $72^{\circ} \mathrm{C}$ and a final incubation for $7 \mathrm{~min}$ at $72^{\circ} \mathrm{C}$. These PCR products were separated by $2 \%$ agarose gel electrophoresis and stained with $0.5 \mu \mathrm{g} / \mathrm{ml}$ ethidium bromide, and the band signals were exposed to ultraviolet light before they were scanned and quantified with a gel image analyzer (GelDoc Quantity One; Bio-Rad, Hercules, CA, USA). Band intensities were quantified and normalized against that of GAPDH.

Western blot analysis. Total retinal protein was extracted from pulverized samples using modified radioimmunoprecipitation (modified RIPA) buffer with a Halt ${ }^{\mathrm{TM}}$ protease and phosphatase inhibitor cocktail (Thermo Scientific, Rockford, IL, USA). The protein concentrations were determined using the method of the Bradford protein assay (Bio-Rad). Each retina served as an individual sample ( $\mathrm{n}=8$ per group). Equal amounts of protein $(20 \mu \mathrm{g} / \mathrm{lane})$ were separated on polyacrylamide gels and then electrotransferred onto nitrocellulose membranes (Amersham, UK). After blocking for $3 \mathrm{~h}$ in Tris-buffered saline with $0.1 \%$ Tween-20 (TBST) and $3 \%$ bovine serum albumin (BSA), membranes were incubated overnight at $4^{\circ} \mathrm{C}$ with the primary antibodies [GAP-43 (1:100, sc-17790), ROCK1 (1:50, sc-17794), ROCK2 (1:50, sc-5561)] in TBST containing $3 \%$ BSA. Membranes were then washed and incubated with alkaline phosphatase-conjugated secondary antibodies in TBST for $2 \mathrm{~h}$ and developed using nitro blue tetrazolium chloride (NBT)/5-bromo-4-chloro-3-indolylphosphate (BCIP) substrate (Promega, Madison, WI, USA). The densities of the bands on the membrane were scanned and analyzed with Image Pro Plus version 6.0 (Media Cybernetics). Twenty-four rat retinas were used for western blot analysis.

RhoA activity assay. Active RhoA was assayed from tissue lysates using a Rho activation assay kit (Upstate Biotechnology, Milton Keynes, UK) following the manufacturer's instructions as described elsewhere (25). Twenty-four rat retinas were used for the RhoA activity assay ( $\mathrm{n}=8$ per group).

Statistical analyses. Data are expressed as means \pm standard deviation, unless otherwise stated. Statistical analyses were performed using SPSS software (IBM SPSS Statistics 19.0). To compare data among the three groups, one-way analysis of variance (ANOVA) followed by post hoc tests was conducted. A P-value $<0.05$ was indicative of a statistically significant result.

\section{Results}

$Y$-39983 promotes $R G C$ survival and axonal regeneration after ONC. The densities of surviving RGCs in the ONC+PBS group and $\mathrm{ONC}+\mathrm{Y}-39983$ group were significantly lower than that in the sham group. However, the density of surviving RGCs in the ONC+Y-39983 group was significantly higher than that in the ONC+PBS group, and the densities of surviving RGCs at $1 / 6,3 / 6,5 / 6$ of the retinal radius in the ONC+Y-39983 group were significantly higher than that at the corresponding regions in the ONC+PBS group, respectively (Fig. 1). The longitudinal optic nerve sections were immunostained for GAP-43 to identify the regenerating axons. The sham group exhibited almost no GAP-43 positive staining and the ONC+PBS group showed few GAP-43-positive axons throughout the crush site (Fig. 2). The ONC+Y-39983 group exhibited numerous GAP-43-positive axons throughout the crush site and distal optic nerve segment (Fig. 2). The number of axons 50, 250 and $500 \mu \mathrm{m}$ from the crush site in the ONC+Y-39983 group was significantly more than that at the corresponding distances in the ONC+PBS group, respectively (Fig. 2).

$Y-39983$ increases GAP-43 protein expression in the retina after $O N C$. The GAP-43 protein expression in the retina was detected by western blotting on day 15 after ONC. Compared with the sham group, the GAP-43 expression in the retina in the $\mathrm{ONC}+\mathrm{PBS}$ and $\mathrm{ONC}+\mathrm{Y}-39983$ groups was significantly increased (Fig. 3). The GAP-43 expression in the retina in the ONC+Y-39983 group was significant higher than that in the ONC+PBS group (Fig. 3), which suggested that Y-39983 upregulates the GAP-43 expression in the retina after ONC. 

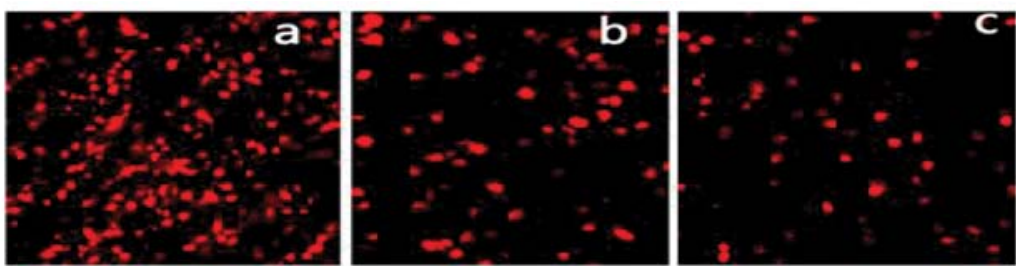

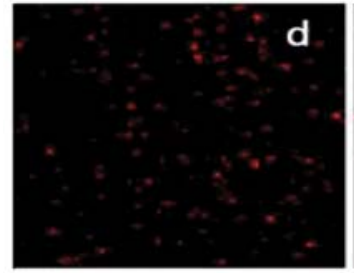

$1 / 6$

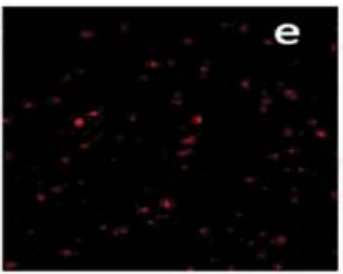

$3 / 6$

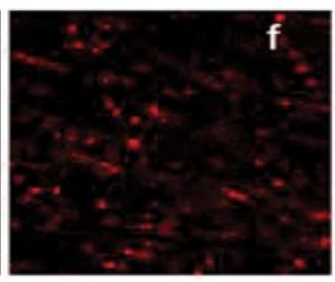

$5 / 6$

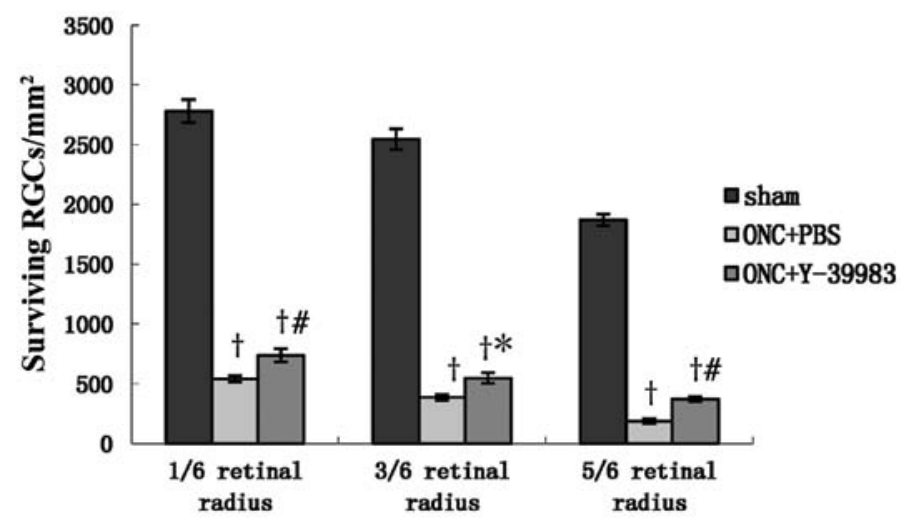

Figure 1. Y-39983 increases RGC survival following ONC. (Top panel) Representative images of the flat-mounted retinas at the corresponding areas (at 1/6, $3 / 6$ and 5/6 of the retinal radius) showing DiI-labeled RGCs in retinas on day 15 after ONC. ONC+Y-39983 group (a-c), ONC+PBS group (d-f). (Bottom panel) Quantitative analysis of the surviving RGCs after ONC in the sham, ONC+PBS, ONC+Y-39983 groups. The densities of surviving RGCs in the ONC+Y-39983 and $\mathrm{ONC}+\mathrm{PBS}$ groups were significantly lower than that in the sham group. The surviving RGC density in the ONC+Y-39983 group was significantly higher than that in the ONC+PBS group. ${ }^{\dagger} \mathrm{P}<0.001$ compared with the sham group; ${ }^{*} \mathrm{P}<0.05,{ }^{\#} \mathrm{P}<0.01$ compared with the ONC+PBS group.
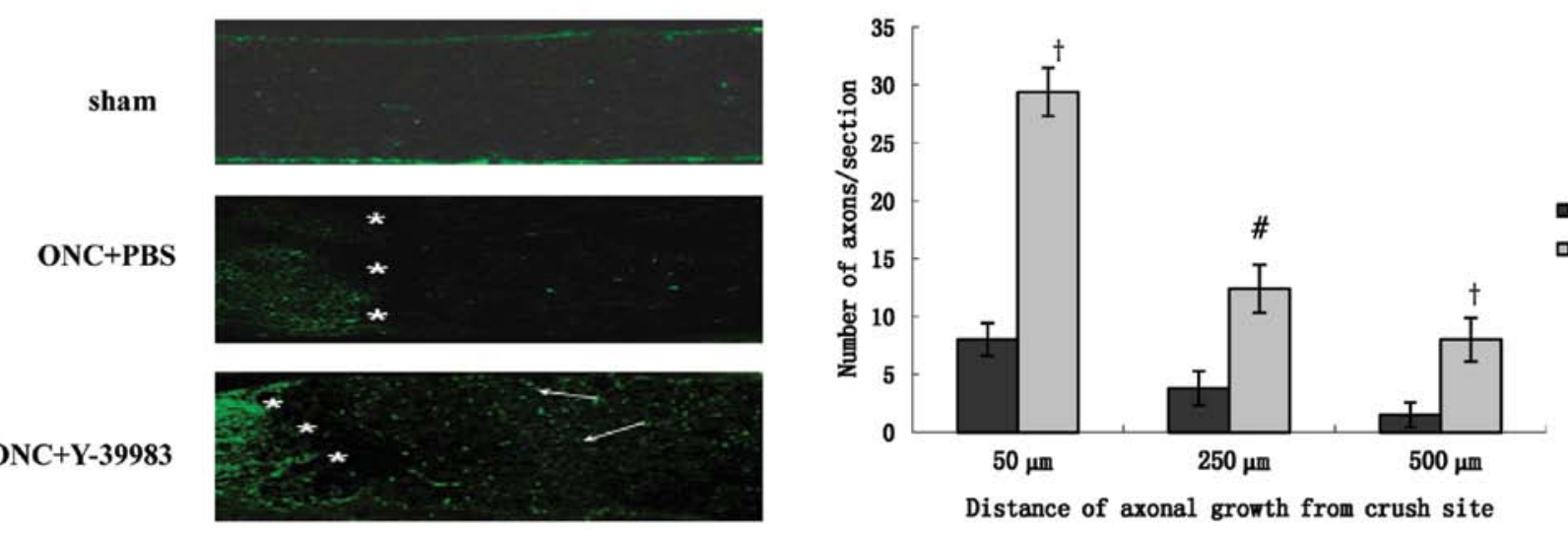

Figure 2. Y-39983 promotes RGC axonal regeneration after ONC. (Left panel) Representative photomicrographs showing the crush site (asterisk) and regenerating axons by GAP-43 immunofluorescence (arrows) on day 15 after ONC in the ONC+PBS and ONC+Y-39983 groups. (Right panel) Quantitative analysis of axon regeneration in the distal optic nerve segment 50,250 and $500 \mu \mathrm{m}$ from the crush site. ${ }^{\#} \mathrm{P}<0.01,{ }^{+} \mathrm{P}<0.001$ compared with the ONC+PBS group.

$Y$-39983 downregulates the expression of active-RhoA, ROCK1 and ROCK2 after ONC. RhoA, ROCK1 and ROCK2 mRNA expression in the retina was detected by RT-PCR on day 15 after ONC. Total RhoA mRNA expression did not differ substantially among the sham, ONC+PBS and ONC+Y-39983 groups (Fig. 4). mRNA expression of ROCK1 and ROCK2 in the ONC+PBS group was significant higher than that in the sham group. Y-39983 downregulated the mRNA expression of ROCK1 and ROCK2 in the retina (Fig. 4).

The protein expression of total-RhoA, active-RhoA, ROCK1 and ROCK2 in the retina or optic nerve was detected by immunohistochemistry, western blotting and affinity 


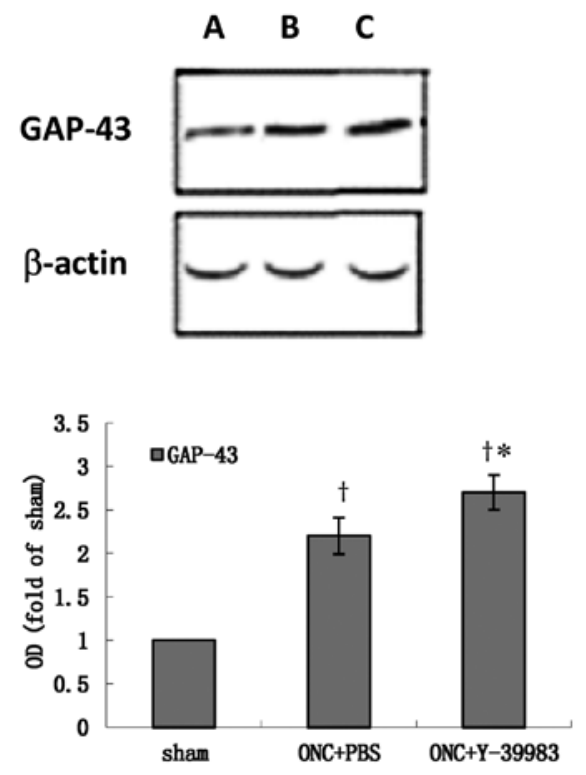

Figure 3. Effect of Y-39983 on the GAP-43 expression in the retina as detected by western blotting after ONC. (Top panel) Representative western blots of GAP-43 in the retina (lane A, sham group; lane B, ONC+PBS group; lane C, ONC+Y-39983 group). Y-39983 resulted in significantly increased GAP-43 expression. (Bottom panel) Quantification of western blot bands from eight independent experiments for GAP-43 protein. ${ }^{\dagger} \mathrm{P}<0.001$ compared with the sham group, ${ }^{*} \mathrm{P}<0.01$ compared with the $\mathrm{ONC}+\mathrm{PBS}$ group.

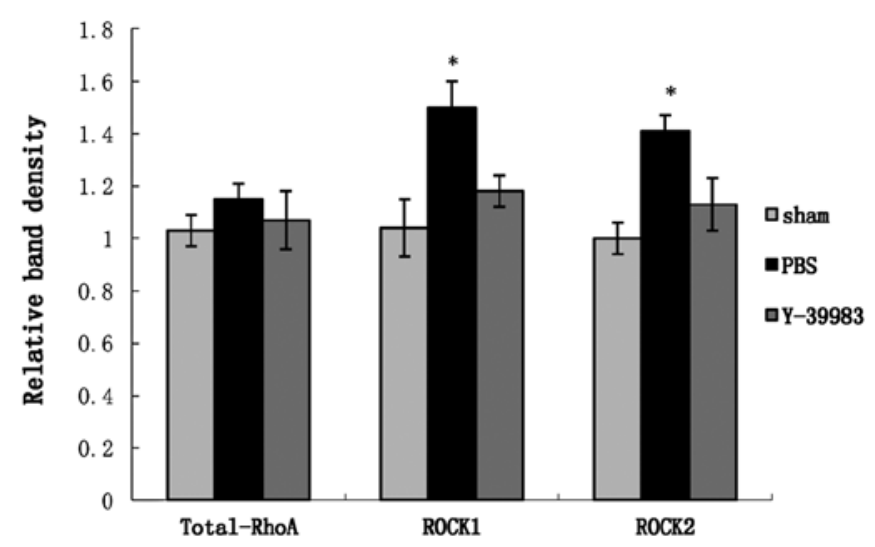

Figure 4. RT-PCR analysis of RhoA, ROCK1 and ROCK2 in the retina by Y-39983 on day 15 after ONC. Total RhoA mRNA expression did not differ substantially in all groups. mRNA expression of ROCK1 and ROCK2 in the ONC+PBS group was significant higher than that in the sham group. Y-39983 downregulated the mRNA expression of ROCK1 and ROCK2 in the retina on day 15 after ONC. ${ }^{*} \mathrm{P}<0.05$ compared with the sham and ONC+Y-39983 groups.

precipitation on day 15 after ONC. The optic nerve in the sham group showed almost no visible active RhoA, ROCK1 and ROCK2 immunofluorescence staining (Fig. 5a-c). The optic nerve in the ONC+PBS group showed strong immunoactivity of active RhoA, ROCK1 and ROCK2 (Fig. 5d-f), while Y-39983 downregulated the immunoactivity of active RhoA, ROCK1 and ROCK2 in the optic nerve after ONC (Fig. 5g-i). Compared with the sham group, the ROCK1 and ROCK2 protein expression in the retina in the ONC+PBS group was significantly increased (Fig. 6), while Y-39983 downregulated
Active fhoA ROCK1 ROCK2
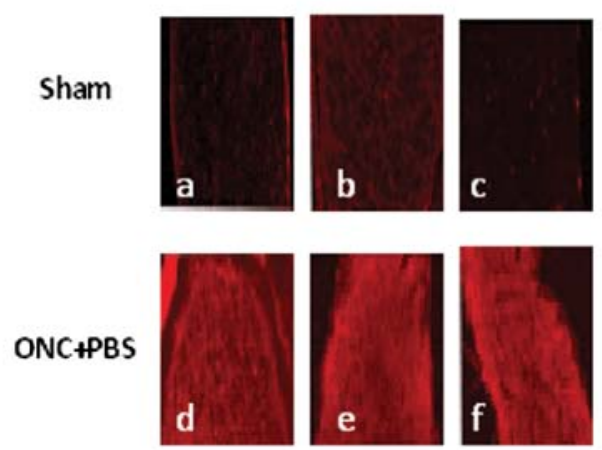

ONC $+Y-39983$
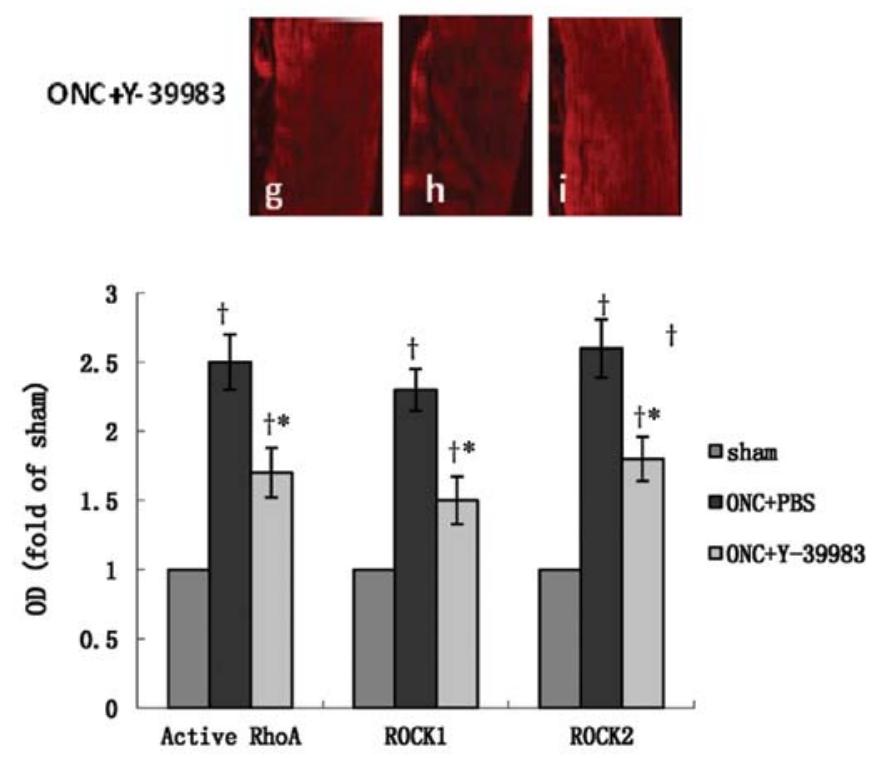

Figure 5. Expression of active-RhoA, ROCK1, ROCK2 in the optic nerve. (Top panel) Representative images of active-RhoA, ROCK1, ROCK2 expression in the optic nerve on day 15 after the ONC. The immunoactivity of active-RhoA in the optic nerve was detected via pull-down assay. Sham group (a-c); ONC+PBS group (d-f); ONC+Y-39983 group (g-i). (Bottom panel) Quantification of immunoactivity from eight independent experiments for active-RhoA, ROCK1 and ROCK2. ${ }^{\dagger} \mathrm{P}<0.05$ compared with the sham group, ${ }^{*} \mathrm{P}<0.05$ compared with the ONC+PBS group.

the ROCK1 and ROCK2 protein expression in the retina on day 15 after ONC (Fig. 6). The total-RhoA protein expression in the retina remained unchanged in all groups as detected by the western blot analysis (Fig. 7). Compared with the sham group, the active-RhoA protein expression in the retina in the ONC+PBS group was significantly increased, while Y-39983 downregulated the active-RhoA protein expression in the retina on day 15 after ONC (Fig. 7).

\section{Discussion}

The multitude of inhibitory molecules present in the lesioned CNS requires a comprehensive strategy with which to identify common inhibitory pathways. The Rho/ROCK pathway represents such a promising target for pharmacological intervention being the convergence point for the signaling of numerous inhibitory substrates. Several ROCK inhibitors, such as Y-27632 $(8,28,29)$, Y-39983 $(17,18,28,29)$, fasudil (28) and dimethylfasudil (28), have been used for the study of 


\section{A B C}
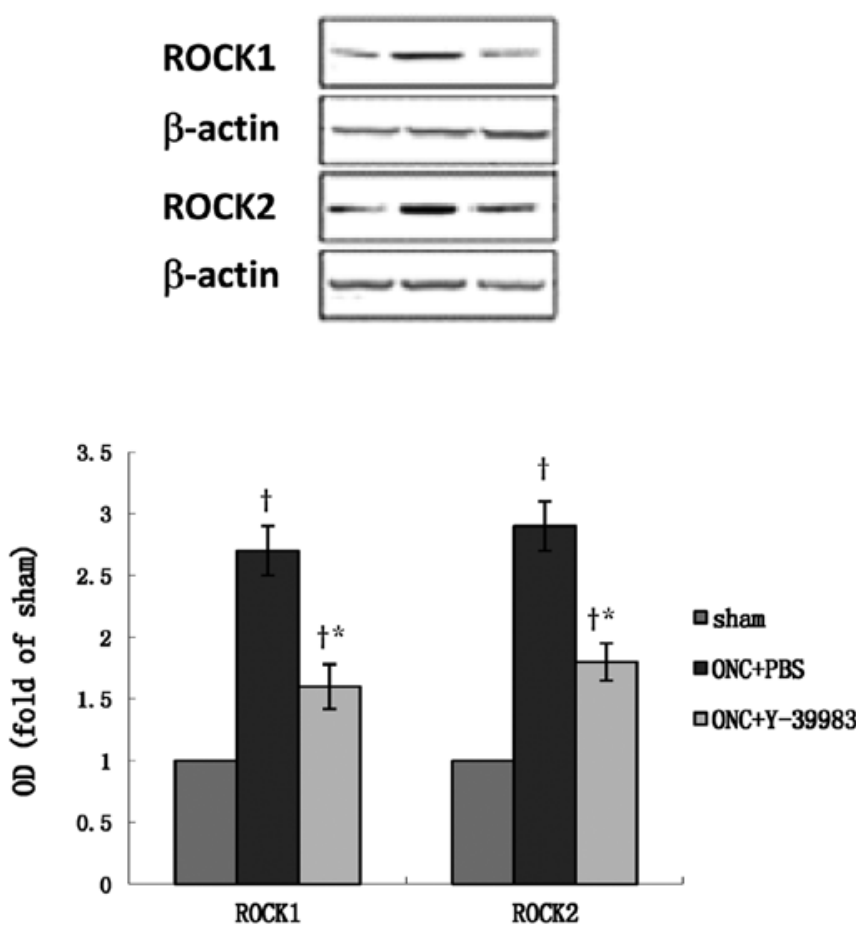

Figure 6. Effect of Y-39983 on ROCK1 and ROCK2 expression in the retina as detected by western blot after ONC. (Top panel) Representative western blots showing the regulation of expression of ROCK1 and ROCK2 in the retina by Y-39983 on day 15 after ONC (lane A: sham group; lane B: ONC+PBS group; lane C: ONC+Y-39983 group). Y-39983 downregulated the protein expression of ROCK1 and ROCK2. (Bottom) Quantification of western blot bands from eight independent experiments for ROCK1 and ROCK2. ${ }^{\dagger} \mathrm{P}<0.05$ compared with the sham group, ${ }^{*} \mathrm{P}<0.05$ compared with the ONC+PBS group.

neuroprotection and axonal regeneration. These inhibitors can promote the neurite outgrowth of RGCs $(17,28)$. In terms of their intensity, the effect of Y-39983 on the promotion of neurite outgrowth was stronger than that of Y-27632, fasudil and dimethylfasudil $(17,28)$. Thus, Y-39983, serving as a candidate of ROCK inhibitors, was chosen for use in this study.

In order to clarify the biological effects of Y-39983, we investigated the effects of Y-39983 on RhoA/ROCK expression during its promotion of axonal regeneration. The present study found that ONC upregulated the expression of active-RhoA, ROCK1 and ROCK2 in the retina or optic nerve. However, Y-39983 downregulated active-RhoA, ROCK1 and ROCK2 expression in the retina or optic nerve on day 15 after ONC and significantly promoted RGC axonal regeneration after ONC.

In addition to its effect of promoting RGC axonal regeneration, Y-39983 could relax isolated ciliary artery (29), increase blood flow in the optic nerve head (18), relax the ciliary muscle and trabecular meshwork (30) and decrease intraocular pressure (IOP) (30-32). Thus, Y-39983 may be a candidate drug not only for lowering IOP but also for increasing blood flow in the optic nerve head in the treatment of glaucoma. Moreover, Y-39983 may have therapeutic potential for axonal regeneration of RGCs in the treatment of diseases presenting with degenerating axons of RGCs including glaucoma.

In summary, we investigated the effects of Y-39983 on RhoA/ROCK expression during its promotion of axonal
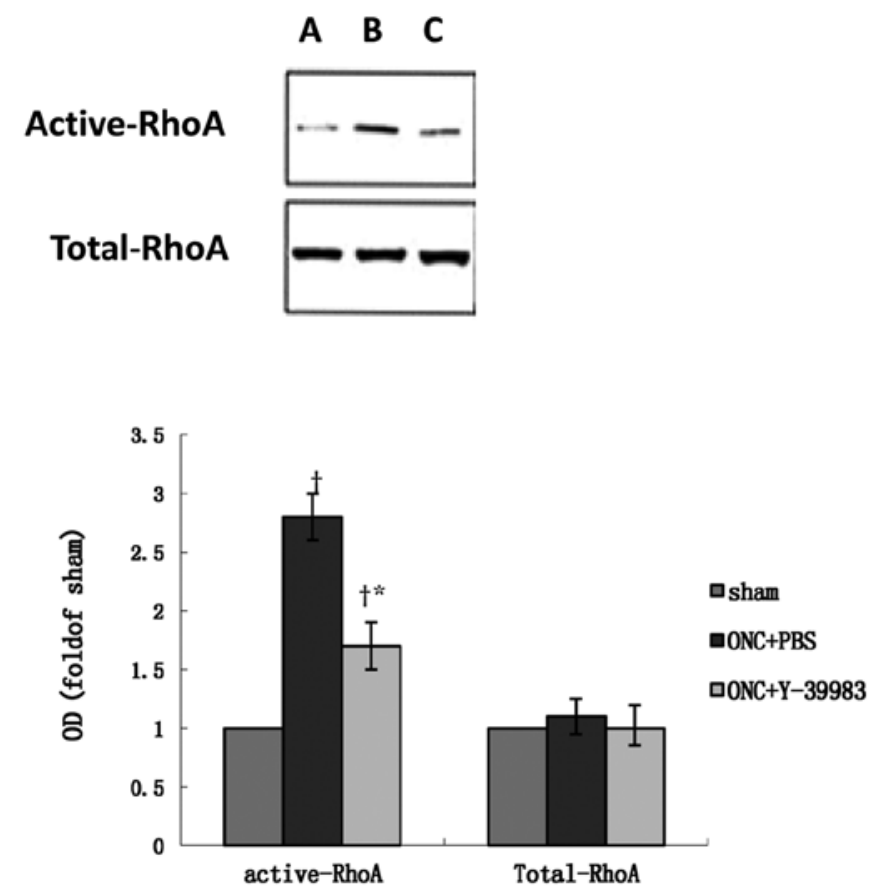

Figure 7. Effect of Y-39983 on total-RhoA and active-RhoA expression in the retina as detected by western blot after ONC. (Top panel) Representative western blots showing the regulation of expression of total-RhoA and activeRhoA in the retina by Y-39983 on day 15 after ONC (lane A, sham group; lane $\mathrm{B}, \mathrm{ONC}+\mathrm{PBS}$ group; lane $\mathrm{C}, \mathrm{ONC}+\mathrm{Y}-39983$ group). Active-RhoA in the retina was detected via GST-RBD binding and immunoblotting with antibody specific to active-RhoA. Total RhoA from the same tissue samples was detected via immunoblotting. The protein expression of total-RhoA did not differ substantially in all groups. Y-39983 downregulated the protein expression of active-RhoA. (Bottom panel) Quantification of western blot bands from eight independent experiments for total-RhoA and active-RhoA protein. ${ }^{\dagger} \mathrm{P}<0.05$ compared with the sham group, ${ }^{*} \mathrm{P}<0.05$ compared with the $\mathrm{ONC}+\mathrm{PBS}$ group.

regeneration. Y-39983 promoted RGC axonal regeneration and downregulated the expression of active-RhoA, ROCK1 and ROCK 2 in the retina or optic nerve on day 15 after ONC. The present study further elucidated the pharmacological effects of Y-39983, making it a promising drug for future therapeutic approaches.

\section{Acknowledgements}

This study was funded by the National Natural Science Foundation of China (nos. 81070728 and 81000373), Shanghai Natural Science Foundation (nos. 08ZR1413900 and 11ZR1422000), Shanghai Leading Academic Discipline Project (no. S30205) and Shanghai 'Science and Technology Innovation Action Plan' Basic Research Key Project (nos. 11JC1407700 and 11JC1407701).

\section{References}

1. Schreyer DJ and Skene JH: Injury-associated induction of GAP-43 expression displays axon branch specificity in rat dorsal root ganglion neurons. J Neurobiol 24: 959-970, 1993.

2. Schaden H, Stuermer CA and Bahr M: GAP-43 immunoreactivity and axon regeneration in retinal ganglion cells of the rat. J Neurobiol 25: 1570-1578, 1994.

3. Domeniconi M and Filbin MT: Overcoming inhibitors in myelin to promote axonal regeneration. J Neurol Sci 233: 43-47, 2005. 
4. Cafferty WB, Duffy P, Huebner E and Strittmatter SM: MAG and OMgp synergize with Nogo-A to restrict axonal growth and neurological recovery after spinal cord trauma. J Neurosci 30: 6825-6837, 2010.

5. Winton MJ, Dubreuil CI, Lasko D, Leclerc N and McKerracher L: Characterization of new cell permeable $\mathrm{C} 3$-like proteins that inactivate Rho and stimulate neurite outgrowth on inhibitory substrates. J Biol Chem 277: 32820-32829, 2002.

6. Dergham P, Ellezam B, Essagian C, Avedissian H, Lubell WD and McKerracher L: Rho signaling pathway targeted to promote spinal cord repair. J Neurosci 22: 6570-6577, 2002.

7. Bertrand J, Winton MJ, Rodriguez-Hernandez N, Campenot RB and McKerracher L: Application of Rho antagonist to neuronal cell bodies promotes neurite growth in compartmented cultures and regeneration of retinal ganglion cell axons in the optic nerve of adult rats. J Neurosci 25: 1113-1121, 2005.

8. Lingor P, Tönges L, Pieper N, Bermel C, Barski E, Planchamp V and Bähr M: ROCK inhibition and CNTF interact on intrinsic signalling pathways and differentially regulate survival and regeneration in retinal ganglion cells. Brain 131: 250-263, 2008.

9. Zhang G, Lehmann HC, Manoharan S, Hashmi M, Shim S, Ming GL, Schnaar RL, Lopez PH, Bogdanova N and Sheikh KA: Anti-ganglioside antibody-mediated activation of RhoA induces inhibition of neurite outgrowth. J Neurosci 31: 1664-1675, 2011.

10. Riento K and Ridley AJ: Rocks: multifunctional kinases in cel behaviour. Nat Rev Mol Cell Biol 4: 446-456, 2003.

11. Olson MF: Applications for ROCK kinase inhibition. Curr Opin Cell Biol 20: 242-248, 2008.

12. Brown ME and Bridgman PC: Myosin function in nervous and sensory systems. J Neurobiol 58: 118-130, 2004.

13. Ishizaki T, Maekawa M, Fujisawa K, Okawa K, Iwamatsu A, Fujita A, Watanabe N, Saito Y, Kakizuka A, Morii N and Narumiya S: The small GTP-binding protein Rho binds to and activates a $160 \mathrm{kDa} \mathrm{Ser} / \mathrm{Thr}$ protein kinase homologous to myotonic dystrophy kinase. EMBO J 15: 1885-1893, 1996.

14. Mehta NR, Nguyen T, Bullen JW Jr, Griffin JW and Schnaar RL: Myelin-associated glycoprotein (MAG) protects neurons from acute toxicity using a ganglioside-dependent mechanism. ACS Chem Neurosci 1: 215-222, 2010.

15. Watanabe M: Regeneration of optic nerve fibers of adult mammals. Dev Growth Differ 52: 567-576, 2010.

16. Ramachandran C, Patil RV, Combrink K, Sharif NA and Srinivas SP: Rho-Rho kinase pathway in the actomyosin contraction and cell-matrix adhesion in immortalized human trabecular meshwork cells. Mol Vis 17: 1877-1890, 2011.

17. Sagawa H, Terasaki H, Nakamura M, Ichikawa M, Yata $T$, Tokita Y and Watanabe M: A novel ROCK inhibitor, Y-39983, promotes regeneration of crushed axons of retinal ganglion cells into the optic nerve of adult cats. Exp Neurol 205: 230-240, 2007.

18. Tokushige H, Waki M, Takayama Y and Tanihara H: Effects of Y-39983, a selective Rho-associated protein kinase inhibitor, on blood flow in optic nerve head in rabbits and axonal regeneration of retinal ganglion cells in rats. Curr Eye Res 36: 964-970, 2011.

19. Yu S, Tanabe T and Yoshimura N: A rat model of glaucoma induced by episcleral vein ligation. Exp Eye Res 83: 758-770, 2006.
20. Leon S, Yin Y, Nguyen J, Irwin N and Benowitz LI: Lens injury stimulates axon regeneration in the mature rat optic nerve. J Neurosci 20: 4615-4626, 2000.

21. Fischer D, Pavlidis M and Thanos S: Cataractogenic lens injury prevents traumatic ganglion cell death and promotes axonal regeneration both in vivo and in culture. Invest Ophthalmol Vis Sci 41: 3943-3954, 2000.

22. Berkowitz BA, Lukaszew RA, Mullins CM and Penn JS: Impaired hyaloidal circulation function and uncoordinated ocular growth patterns in experimental retinopathy of prematurity. Invest Ophthalmol Vis Sci 39: 391-396, 1998.

23. Yin $Y$, Cui Q, Li Y, Irwin N, Fischer D, Harvey AR and Benowitz LI: Macrophage-derived factors stimulate optic nerve regeneration. J Neurosci 23: 2284-2293, 2003.

24. Dubreuil CI, Winton MJ and McKerracher L: Rho activation patterns after spinal cord injury and the role of activated Rho in apoptosis in the central nervous system. J Cell Biol 162: 233-243, 2004.

25. Ahmed Z, Suggate EL, Brown ER, Dent RG, Armstrong SJ, Barrett LB, Berry M and Logan A: Schwann cell-derived factorinduced modulation of the NgR/p75NTR/EGFR axis disinhibits axon growth through CNS myelin in vivo and in vitro. Brain 129: $1517-1533,2006$.

26. Nakamura M, Nagano T, Chikama $T$ and Nishida T: Role of the Small GTP-binding protein Rho in epithelial cell migration in the rabbit cornea. Invest Ophthalmol Vis Sci 42: 941-947, 2001.

27. Kim BK, Kim HM, Chung KS, Kim DM, Park SK, Song A, Won KJ, Lee K, Oh YK, Lee K, Song KB, Simon JA, Han G and Won M: Upregulation of RhoB via c-Jun N-terminal kinase signaling induces apoptosis of the human gastric carcinoma NUGC-3 cells treated with NSC12618. Carcinogenesis 32: 254-261, 2011.

28. Lingor P, Teusch N, Schwarz K, Mueller R, Mack H, Bähr M and Mueller BK: Inhibition of Rho kinase (ROCK) increases neurite outgrowth on chondroitin sulphate proteoglycan in vitro and axonal regeneration in the adult optic nerve in vivo. J Neurochem 103: 181-189, 2007.

29. Watabe H, Abe S and Yoshitomi T: Effects of Rho-associated protein kinase inhibitors Y-27632 and Y-39983 on isolated rabbit ciliary arteries. Jpn J Ophthalmol 55: 411-417, 2011.

30. Nakajima E, Nakajima T, Minagawa Y, Shearer TR and Azuma M: Contribution of ROCK in contraction of trabecular meshwork: proposed mechanism for regulating aqueous outflow in monkey and human eyes. J Pharm Sci 94: 701-708, 2005.

31. Tokushige H, Inatani M, Nemoto S, Sakaki H, Katayama K, Uehata $\mathrm{M}$ and Tanihara $\mathrm{H}$ : Effects of topical administration of y-39983, a selective rho-associated protein kinase inhibitor, on ocular tissues in rabbits and monkeys. Invest Ophthalmol Vis Sci 48: 3216-3222, 2007

32. Whitlock NA, Harrison B, Mixon T, Yu XQ, Wilson A, Gerhardt B, Eberhart DE, Abuin A and Rice DS: Decreased intraocular pressure in mice following either pharmacological or genetic inhibition of ROCK. J Ocul Pharmacol Ther 25: 187-194, 2009. 